\title{
Modifying Young's Modulus in DEM Simulations Based on Distributions of Experimental Measurements
}

\author{
Jon T. Van Lew ${ }^{\mathrm{a}, *}$, Yi-Hyun Park ${ }^{\mathrm{b}}$, Alice Ying ${ }^{\mathrm{a}}$, Mohamed Abdou \\ ${ }^{a}$ UCLA, MAE Department, 44-114 Engineering IV, 420 Westwood Plaza, Los Angeles, CA 90095-1597, USA \\ ${ }^{b}$ National Fusion Research Institute, Daejeon, Republic of Korea
}

\begin{abstract}
The discrete element method, as currently employed by members of the fusion community, is rooted on the assumption that each pebble is a perfectly elastic material that obeys Hertz's theory for normal interaction. This assumption impacts the magnitude of inter-particle forces predicted by the models. We scrutinize the Hertzian assumption with single-pebble crush experiments with carefully recorded force-displacement responses and compare them to the non-linear forces predicted by a Hertzian pebble with bulk properties reported in literature. We found each pebble generally has a non-linear force response but with varying levels of stiffness that qualitatively matched the curves from Hertz theory. Assuming Hertzian interaction, we backed-out an elastic modulus for each pebble. We define a softening coefficient, $\kappa$, as the ratio of the pebble's elastic modulus to the sintered bulk value from literature. After determining the $\kappa$ value for every pebble in our batch, we discovered a probability distribution for different batches. The distribution is attributed to the varying micro-structure of each pebble. We incorporate the results into our DEM algorithms, distributing $\kappa$ values at random to pebbles satisfying the probability curves of experiments. DEM simulations of pebble beds in oedometric compression are carried out to determine macroscopic responses of stress-strain, contact force distributions at maximum stress, and a prediction of pebbles crushing at that point. In all cases studied here, the pebble beds with modified Young's modulus had smaller overall contact forces and fewer predicted crushed pebbles.
\end{abstract}

Keywords:

\section{Introduction}

The discrete element method (DEM) is used by many ceramic breeder researchers to model the interaction of individual pebbles in an ensemble in an effort to obtain a more detailed understanding of pebble beds than is possible with experimental measurements of effective properties; recent examples can be found in the work of Refs. [1-6]. A major assumption in the DEM formulation is that each pebble acts perfectly elastically and adheres to Hertz theory for contacting spheres. With Hertz theory, one finds contact forces as a simple function of: the virtual overlap between two objects, the Young's modulus of the contacting material (and

\footnotetext{
*Corresponding author

Email address: jtvanlew@fusion.ucla.edu (Jon T. Van Lew )

Preprint submitted to Fusion Engineering and Design
}

Poisson ratio), and radii of the two. In past studies, the Young's modulus of the ceramic materials used in DEM simulations was taken from historical data, for instance lithium metatitanate from Ref. [7].

Based on observations of experimental data from single pebble crush data, in this study we propose a new method of obtaining the Young's modulus for a batch of ceramic pebbles and show examples of the implementation of the Young's modulus in a DEM framework in the cases when historical values from literature are not appropriate.

\section{Motivation}

In the study of individual pebble crush force, the force-travel response curves of ceramic materials consistently exhibit distributions in the stiffness of the pebbles. For example, see the results of different lithium ceramic pebbles in Fig. 1. For

June 2, 2015 
DEM studies, it is claimed that interaction between these pebbles is well-represented by Hertz theory, as given in Eq. 1.

With Hertz theory, we can relate the normal contact force in terms of virtual overlap between two spheres,

$$
F_{n}=\frac{4}{3} E^{*} \sqrt{R^{*}} \delta^{3 / 2}
$$

where $\delta$ is the overlap between contacting spheres, $\frac{1}{E^{*}}=\frac{1-\nu_{i}^{2}}{E_{i}}+\frac{1-\nu_{j}^{2}}{E_{j}}$, and $\frac{1}{R^{*}}=\frac{1}{R_{i}}+\frac{1}{R_{j}}$. Because the contact force is directly proportional to the pair Young's modulus, $E^{*}$, an accurate value of pebble Young's modulus is directly related to the accuracy of DEM computations of contact force. For brevity, we do not include many details on DEM, but refer the reader to the original paper by Cundall and Strack upon which all modern models are based.[8]

We rewrite Eq. 1 to apply it to experimental measurements of individual pebbles being crushed between to anvils. For a pebble of diameter $d_{p}$, where we measure the travel of one anvil as $s$, The Hertz force on the pebble is,

$$
F_{n}=\frac{1}{3} E^{*} \sqrt{d_{p} s^{3}}
$$

and $\frac{1}{E^{*}}=\frac{1-\nu_{p}^{2}}{E_{p}}+\frac{1-\nu_{a}^{2}}{E_{a}}$. Where the subscript $p$ refers to pebbles and $a$ the anvils.

From Eq. 2, we see that standard Hertz theory, wherein a single value for Young's modulus is used, is not appropriate for pebbles studied in ceramic breeders. If single values of $E_{p}$ and $\nu_{p}$ are employed, then variation in pebble diameters can not alone explain the variation of curves of Fig. 1.

\section{Analysis}

\subsection{Experimental Setup}

The results presented here are based on two batches of pebbles, one each of $\mathrm{Li}_{4} \mathrm{SiO}_{4}$ and $\mathrm{Li}_{2} \mathrm{TiO}_{3}$. The $\mathrm{Li}_{4} \mathrm{SiO}_{4}$ pebbles were provided by the Karlsruhe Institute of Technology; thirty-one pebbles were used from the batch. Details of the material can be found in Ref. [9]. The $\mathrm{Li}_{2} \mathrm{TiO}_{3}$ pebbles were provided by The National Fusion Research Institute of South Korea; forty-two pebbles were tested for this data set. Details of the manufacturing process and material properties can be found in Ref. [10]. The experiments were run at room temperature with uncontrolled humidity.

\subsection{Elasticity Reduction Factor}

We propose to explain the behavior of individual pebbles (as in Fig. 1) with an assumption that the production technique yields pebbles with slightly different internal structures. The differences in internal structure then cause the pebble to have a different apparent modulus of elasticity; which will vary from some strong limit value. The strong value is the elastic modulus of highly sintered pellets reported in literature for the material, $E_{\text {bulk }}$. Assuming this strong value is the upper limit, imperfections in the pebbles will lead only to a reduction, or softening of the pebble. To quantify the deviation from the bulk, we introduce $\kappa$, defining it as a softening coefficient,

$$
\kappa=\frac{E_{\mathrm{peb}}}{E_{\mathrm{bulk}}}
$$

where $\kappa \in[0,1]$.

If each pebble has a unique $\kappa$ value, this would quantify the spread in elastic responses seen in the experiments. The value is found by assuming that the pebbles are, in fact, behaving in a Hertzian manner, allowing us to back-out its $\kappa$ value, or in other words the unique $E^{*}$ of that pebble by finding a best fit to the experimental curves.

From room temperature, we take the sintered pebble value for these $\mathrm{Li}_{4} \mathrm{SiO}_{4}$ pebbles to be $E_{\text {bulk }}=90 \mathrm{GPa}$ and $E_{\text {bulk }}=124 \mathrm{GPa}$ for $\mathrm{Li}_{2} \mathrm{TiO}_{3}$ pebbles. Then we iterate over all values of $\kappa \in[0,1]$ and compare the Hertzian response to that pebble's force-displacement curve.

The data of Fig. 1a is fit in the manner described and the pebbles are all plotted against Hertzian curves with their own unique modified Young's modulus in Fig. 2a. The modified Hertzian curves with apparent Young's modulus fits well with most of the pebbles' curves. The distribution of $\kappa$ is given in Fig. 2b. Similar data is obtained for the pebbles of Fig. 1b but the results are omitted for brevity.

One of the uses of DEM simulations is prediction of pebble cracking in an ensemble based on knowledge of the interaction forces. If we are overpredicting the contact forces based on incorrect material properties input into the model, we are going to over-predict the extent of pebble cracking as well. An accurate description of the material properties is an important feature for ceramic breeder designers. 


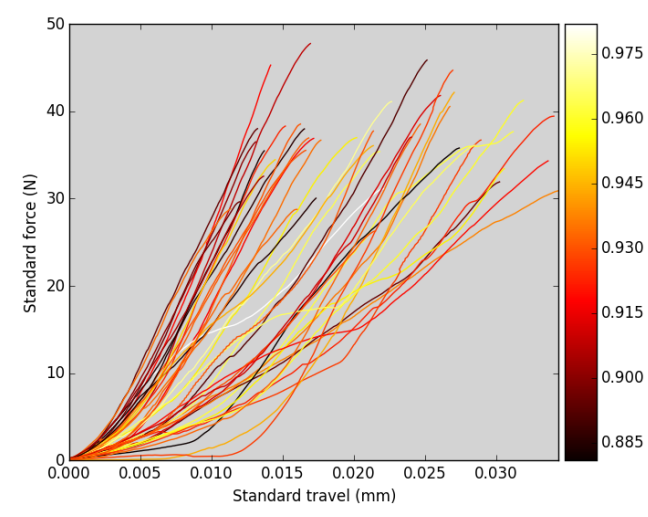

(a) $\mathrm{Li}_{2} \mathrm{TiO}_{3}$ pebbles of $d_{p}=1 \mathrm{~mm}$.

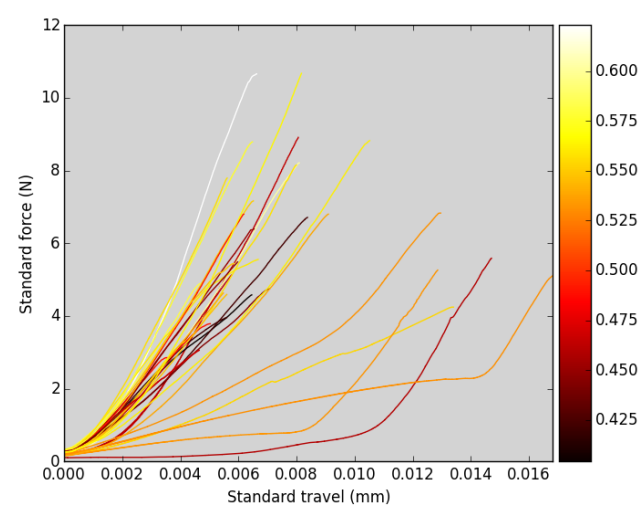

(b) $\mathrm{Li}_{4} \mathrm{SiO}_{4}$ pebbles of $d_{p}=0.5 \mathrm{~mm}$.

Figure 1: [color online] Force-travel responses of various lithiated ceramic pebbles of disparate diameters show similar behavior in distributions of responses. The colormaps differentiate the pebbles by diameter (in millimeters).

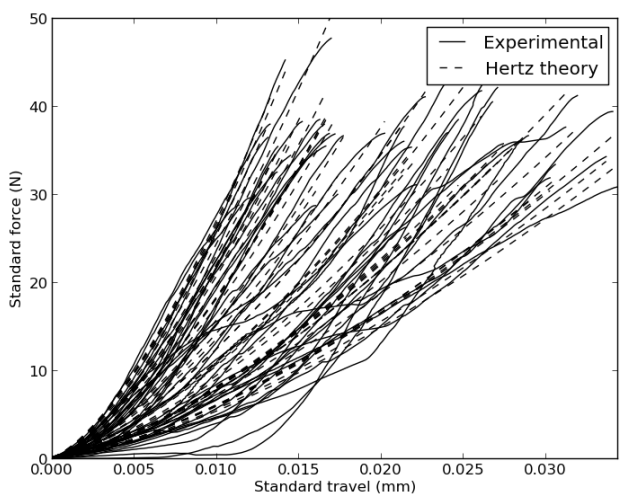

(a) Experimental responses (solid) and fit curves of Hertzian equivalent with apparent Young's modulus (dashed).

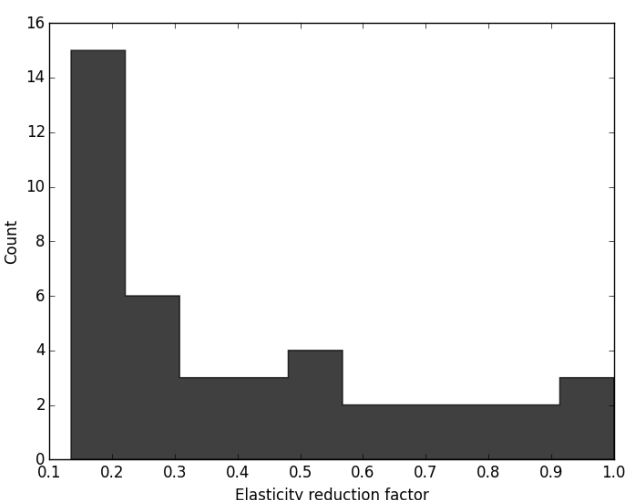

(b) Distribution of elasticity reduction value, $\kappa$, for the pebbles in this batch of lithium metatitanate. This distribution is modeled as a Weibull distribution function in DEM simulations.

Figure 2: An apparent Young's modulus is found for each pebble and the distribution of reduction factor, $\kappa$, shows the quantity of reduction of the stiffness of the pebbles from the value found in literature.

\subsection{Pebble Crushing Predictions}

Along with proper material properties, we present a relationship to translate between the experimental data of crush force to a value that can be applied to DEM simulations. We integrate the Hertzian force (given in Eq. 1) along the contact overlap to find the strain energy, $W_{\epsilon}$, of a given contact,

$$
W_{\epsilon}=\int_{0}^{\delta_{c}} \frac{4}{3} E^{*} \sqrt{R^{*}} \delta^{\prime 3 / 2} \mathrm{~d} \delta^{\prime}
$$

We call the strain energy of the pebble com- pressed between platens as the lab strain energy, $W_{\epsilon, l}$. The strain energy of two particles in contact in the bed will be $W_{\epsilon, b}$. The assumption is made that, if each contact interaction is integrated to the proper critical overlap, the strain energies will be equal at that contact. The integral of Eq. 4 is evaluated for a contact in the ensemble and set equal to the critical lab value as,

$$
W_{\epsilon, l}=W_{\epsilon, b}=\frac{8}{15} E_{b}^{*} \sqrt{R_{b}^{*}} \delta_{c, b}^{5 / 2}
$$

which allows one to solve for the critical interacting pebble bed overlap as a function of the lab strain 
energy,

$$
\delta_{c, b}=\left[\frac{15 W_{\epsilon, l}}{8 E_{b}^{*} \sqrt{R_{b}^{*}}}\right]^{2 / 5}
$$

Finally, this overlap is reinserted to the Hertz force of Eq. 1 to find the critical force (crush force) of the interacting particles in the numeric ensemble as a function of the critical strain energy measured in the lab. The relationship is,

$$
F_{c, b}=C E_{b}^{* 2 / 5} R_{b}^{* 1 / 5} W_{\epsilon, l}^{3 / 5}
$$

where $C=\frac{4}{3}\left(\frac{15}{8}\right)^{3 / 5}$. $W_{\epsilon, l}$ is the measured strain energy up to the point of crushing from experiments. This value follows a probability distribution and therefore naturally imparts a distribution shape to the $F_{c, b}$ prediction of Eq. 7 . We use the above prediction to determine how many pebbles would crush in the ensemble based on the external load of the bed. The methodology of this crush prediction, based on a critical contact force, stands in contrast to a similar approach reported by Zhao et $a l$ where they based crush prediction on the total strain energy of a pebble summed among all contacts acting upon it.[11] This prediction is based on the results of Russell et al where they showed that the mechanics of a contact between spheres in an ensemble are highly localized and therefore the internal stresses of the contact are independent of the number or magnitude of neighboring contacts.[12]

\subsection{Numerical Experiments Setup}

We model our pebble beds undergoing a standard stress-controlled uniaxial compression up to $6 \mathrm{MPa}$. We will measure the macroscopic stressstrain for some parametrically varied pebble beds and compare the curves. At the moment of maximum stress, we can investigate the differences in contact forces of those pebble beds and the extent of crushing predicted in the bed.

Our pebble ensemble is composed of $0.5 \mathrm{~mm}$ diameter lithium orthosilicate pebbles. The pebble beds are initiated and packed in the same manner as Ref. [6]. There are two main bed groups. Set A: the first set of three beds (A.1-3) contain a single type of pebble with $E=90 \mathrm{GPa}$. Set B: the second set of four beds (B.1-4) contain ten types of pebbles with their Young's modulus assigned in a discrete, random way to satisfy the distribution seen from experimental data. For the DEM study, we fit to lithium orthosilicate pebbles where the average stiffness was $\bar{E}=49 \mathrm{GPa}$. The description of
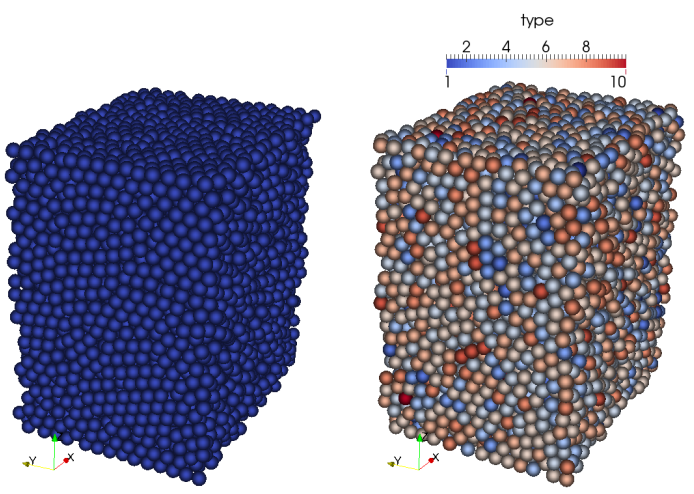

Figure 3: [color online] On the left, set A, a pebble bed with a single type, of $E=90 \mathrm{GPa}$. On the right, set $\mathrm{B}$, is a pebble bed with 10, randomly distributed types; each type corresponds to a reduced, apparent Young's modulus as derived from experimental data.

the two sets of pebble beds is visually represented in Fig. 3. The pebble bed geometry was also the same used in the study of Ref. [6]: two virtual walls obeying the Hertz force equation for interaction with pebbles with the limit of the flat wall going to infinite radius and mass with a Young's modulus set to $220 \mathrm{GPa}$ to mimic a piece of steel, located at $x_{\lim }= \pm 20 R_{p}$; periodic boundaries at the limits of $y_{\text {lim }}= \pm 15 R_{p}$; and a total of 8000 pebbles packed into the volume to an approximate height of $z_{\lim }=20 R_{p}$.

Among both sets, a parametric study was done on pebble radius and coefficient of friction. The radii of pebbles in beds A.1, A.2, B.1, and B.2 were constant at $R_{p}=0.25 \mathrm{~mm}$. The radii of pebbles in beds A.3, B.3, and B.4 followed a Gaussian distribution about $\bar{R}_{p}=0.25 \mathrm{~mm}: \mu_{d}=R_{p}$ and $\sigma_{d}=\frac{R_{p}}{15}$. The coefficient of friction was set at $\mu=0.2$ for beds A.1, A.3, B.1, and B.3; the coefficient of friction was $\mu=0.3$ for beds A.2, B.2, and B.4.

\section{Results}

A constant-velocity, uniaxial compression was applied to the pebble beds. A single cycle peaking at $6 \mathrm{MPa}$ was used on all the beds. The macroscopic measurements of stress-strain are shown for all the pebble beds in Fig. 4.

Intuitively, the pebble beds with smaller Young's modulus (with circle markers) are more compliant to external loads. The result is true regardless of the coefficient of friction or distribution of pebble 


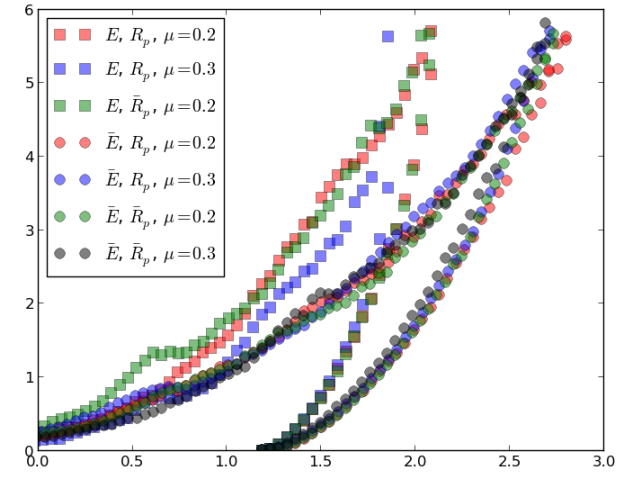

Figure 4: [color online] Stress-strain responses of pebble beds with: squares, constant Young's modulus; and circles, Gaussian distribution of Young's modulus. The constant Young's modulus beds all had much firmer responses for all parametric cases studied here.

radius studied here. Group B moved to an average strain of about $2.6 \%$ at $6 \mathrm{MPa}$, by comparison the beds of Group A only had strained $1.9 \%$, on average, to reach the same stress. Among the beds of each group, pebble beds with constant radius pebbles behaved virtually the same as similar pebble beds with a Gaussian distribution on radius. An increase in the coefficient of friction had a moderate impact on the overall stress-strain response.

The parametric study here shows that the largest contributor to stress-strain response is the Young's modulus. The coefficient of friction and radius distribution had comparatively insignificant influence. A pebble bed geometry more directly comparable to oedometric compression experiments should be used to allow direct comparison and validation of the numerical models.

At the point of peak stress for each bed, we use DEM results to visualize the distribution of contact forces among all pebbles in the ensemble. A plot of the probability distributions of all the beds together, Fig. 5, shows that the majority of the contacts in all the beds are equally small. There are a few overall trends we observe from the results however. The pebble beds with the constant Young's modulus are always higher for their comparable version with distributed Young's modulus. For pebble beds with comparable Young's modulii and radii, higher coefficients of friction generally have higher peak contact forces. Pebble beds' radius distributions have much less impact on peak contact forces than either coefficient of friction or Young's modu-

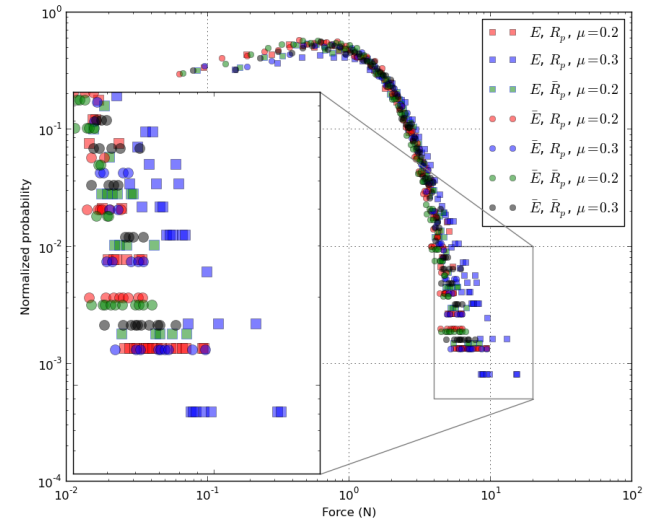

Figure 5: [color online] Probability distribution of contact forces in all the pebble beds studied here. Elastic moduli value is the largest contributor to higher peak contact forces among pebbles.

lus. Another method of comparing overall contact force distributions is to consider predictions on pebble cracking which assigns a strength value at random to pebbles in the bed, according to Eq. 7. At the point of maximum stress, this is done and the results are shown in Tab. 1.

While overall the predicted number of broken pebbles is small, we compare similar parameteric pebble beds and in each case pebble beds with modified Young's modulus overall predict smaller percentages of broken pebbles. Pebble crushing is a major topic for the overall evaluation of the feasibility of ceramic pebble beds in fusion reactors. This study reveals that past DEM work on pebble crushing was likely over-predicting the extent of crushing if the Young's modulus used in the study was much larger than the realistic response of individual pebbles.

\section{Conclusions}

Models based on the discrete element method have received considerable attention by ceramic breeder researchers. The method is attractive as it is based on material properties and forgoes many of the assumptions necessary in empirical equations of effective properties for continuum models. Variation in production techniques for ceramic pebbles lead to changes in ceramic microstructure, as evident in the wide distribution of crush loads reported in this and past studies, e.g. Refs. $[13,14]$. By the same token, the different microstructures should 
Table 1: Comparisons for the two styles of Young's modulii used in the study.

\begin{tabular}{lll}
\hline Bed label & Parameters & Predicted crushed \\
& & \\
\hline A.1 & $E, R_{p}, \mu=0.2$ & 0.3 \\
\hline A.2 & $E, R_{p}, \mu=0.3$ & 1.0 \\
\hline A.3 & $E, \bar{R}_{p}, \mu=0.2$ & 0.9 \\
\hline B.1 & $\bar{E}, R_{p}, \mu=0.2$ & 0.6 \\
\hline B.2 & $\bar{E}, R_{p}, \mu=0.3$ & 0.8 \\
\hline B.3 & $\bar{E}, \bar{R}_{p}, \mu=0.2$ & 0.4 \\
\hline B.4 & $\bar{E}, \bar{R}_{p}, \mu=0.3$ & 0.7 \\
\hline
\end{tabular}

also lead to variation in Young's modulii. However, up to now, values of Young's modulii used in numerical models are taken from values measured for large sintered pellets of ceramic materials. Based on single pebble experiments and the application of Hertz theory, a technique for introducing a modified Young's modulus into DEM models has been proposed here. DEM simulations show the impact of modified pebble elasticity on both macroscopic measurements of stress-strain curves as well as mesoscopic measures of inter-pebble contact force - with major implications for prediction of pebble crushing in ceramic pebble beds. The models applying the softening coefficient, $\kappa$, predict more compliant pebble beds and smaller peak contact forces in beds and thus fewer crushed pebbles.

\section{Acknowledgments}

This work was performed with joint support from US Department of Energy, Office of Fusion Energy Sciences, under Grant No. DE-FG02-86ER52123, and Task Agreement between UCLA-NFRI for Cooperation on R\&D for Fusion Nuclear Science to Expedite the Realization of Magnetic Fusion Energy.

[1] Z. An, A. Ying, M. A. Abdou, Numerical characterization of thermo-mechanical performance of breeder pebble beds, Journal of Nuclear Materials 367-370 (2007) 1393-1397.

[2] Z. Lu, Numerical Modeling and Experimental Measurement of the Thermal and Mechanical Properties of Packed Beds, Ph.D. thesis, University of California Los Angeles, 2000.
[3] S. Zhao, Multiscale Modeling of Thermomechanical Properties of Ceramic Pebbles, Ph.D. thesis, Karlsruhe Institute of Technology, 2010.

[4] Y. Gan, M. Kamlah, Discrete element modelling of pebble beds: With application to uniaxial compression tests of ceramic breeder pebble beds, Journal of the Mechanics and Physics of Solids 58 (2010) 129-144.

[5] R. K. Annabattula, Y. Gan, S. Zhao, M. Kamlah, Mechanics of a crushable pebble assembly using discrete element method, Journal of Nuclear Materials 430 (2012) 90-95.

[6] J. T. Van Lew, A. Ying, M. A. Abdou, A discrete element method study on the evolution of thermomechanics of a pebble bed experiencing pebble failure, Fusion Engineering and Design 89 (2014) 1151-1157.

[7] P. J. Gierszewski, Review of properties of lithium metatitanate, Fusion Engineering and Design 39-40 (1998) 739-743.

[8] P. A. Cundall, O. D. L. Strack, A discrete numerical model for granular assemblies, Geotechnique 29 (1979) 47-65.

[9] J. Reimann, E. Arbogast, M. Behnke, S. Müller, K. Thomauske, Thermomechanical behaviour of ceramic breeder and beryllium pebble beds, Fusion Engineering and Design 49-50 (2000) 643-649.

[10] Y.-H. Park, S. Cho, M.-Y. Ahn, Fabrication of Li2TiO3 pebbles using PVA-boric acid reaction for solid breeding materials, Journal of Nuclear Materials 455 (2014) 106-110.

[11] S. Zhao, Y. Gan, M. Kamlah, T. Kennerknecht, R. Rolli, Influence of plate material on the contact strength of $\mathrm{Li} 4 \mathrm{SiO} 4$ pebbles in crush tests and evaluation of the contact strength in pebble-pebble contact, Engineering Fracture Mechanics 100 (2013) 28-37.

[12] A. R. Russell, D. Muir Wood, M. Kikumoto, Crushing of particles in idealised granular assemblies, Journal of the Mechanics and Physics of Solids 57 (2009) 12931313.

[13] S. Zhao, Y. Gan, M. Kamlah, Spherical ceramic pebbles subjected to multiple non-concentrated surface loads, International Journal of Solids and Structures 49 (2012) 658-671.

[14] D. Mandal, D. Sathiyamoorthy, V. G. Rao, Preparation and characterization of lithium-titanate pebbles by solid-state reaction extrusion and spherodization techniques for fusion reactor, Fusion Engineering and Design 87 (2012) 7-12. 